\title{
Deciding Bit-Vector Arithmetic with Abstraction ${ }^{\star}$
}

\author{
Randal E. Bryant ${ }^{1}$, Daniel Kroening ${ }^{2}$, Joël Ouaknine ${ }^{3}$, Sanjit A. Seshia ${ }^{4}$, \\ Ofer Strichman ${ }^{5}$, and Bryan Brady ${ }^{4}$ \\ ${ }^{1}$ Carnegie Mellon University, Pittsburgh \\ ${ }^{2}$ ETH Zürich \\ ${ }^{3}$ Oxford University Computing Laboratory \\ ${ }^{4}$ University of California, Berkeley \\ 5 The Technion, Haifa
}

\begin{abstract}
We present a new decision procedure for finite-precision bitvector arithmetic with arbitrary bit-vector operations. Our procedure alternates between generating under- and over-approximations of the original bit-vector formula. An under-approximation is obtained by a translation to propositional logic in which some bit-vector variables are encoded with fewer Boolean variables than their width. If the underapproximation is unsatisfiable, we use the unsatisfiable core to derive an over-approximation based on the subset of predicates that participated in the proof of unsatisfiability. If this over-approximation is satisfiable, the satisfying assignment guides the refinement of the previous underapproximation by increasing, for some bit-vector variables, the number of Boolean variables that encode them. We present experimental results that suggest that this abstraction-based approach can be considerably more efficient than directly invoking the SAT solver on the original formula as well as other competing decision procedures.
\end{abstract}

\section{Introduction}

Decision procedures for quantifier-free fragments of first-order logic find widespread use in hardware and software verification. Current uses of decision procedures fall into one of two extremes. At one end, a Boolean satisfiability solver is directly employed as the decision procedure, with systems modeled at the bit-level. Sample applications of this kind include bounded model checking 12 and SAT-based program analysis [3]. At the other extreme, verifiers use decision procedures that reason over arbitrary-precision abstract types such as the integers and reals $(\mathbb{Z}$ and $\mathbb{R})$.

In reality, system descriptions are best modeled with a level of precision that is somewhere in between. System descriptions are usually at the word-level; i.e., they use finite-precision arithmetic and bit-wise operations on bit-vectors. The direct use of a SAT solver as cited earlier (also known as "bit-blasting") is the

\footnotetext{
* B. Brady, R. E. Bryant, and S. A. Seshia were supported in part by SRC contract 1355.001 and by the MARCO Gigascale Systems Research Center.
} 
conceptually simplest way to implement a bit-vector decision procedure even though it ignores higher-level structure present in the original decision problem.

However, the bit-blasting approach can be too computationally expensive in practice (see, for example, 4]). There is therefore a pressing need for better decision procedures for bit-vector arithmetic.

Contribution. We present a decision procedure for quantifier-free bit-vector arithmetic that uses automatic abstraction-refinement. This procedure is now implemented in the verification system UCLID, and we shall call it by this name from hereon. Given an input bit-vector formula $\phi$, UCLID first builds an underapproximation $\phi$ from $\phi$ by restricting the number of Boolean variables used to encode each bit-vector variable (see details of this encoding in Section 3.1). The reduced formula is typically much smaller and easier to solve. If $\phi$ is satisfiable, so is $\phi$, and the algorithm terminates. In case the Boolean formula is found to be unsatisfiable, the SAT solver is able to output a resolution proof of this fact, from which the unsatisfiable core used in this proof can be extracted. Using this core, an over-approximation $\bar{\phi}$ is built. This over-approximation uses the full set of bits of the original vectors, but only a subset of the constraints. This subset is determined by examining the unsatisfiable core of $\phi$. If $\bar{\phi}$ is unsatisfiable, so is $\phi$, and UCLID terminates. Otherwise, the algorith $\bar{m}$ refines the under-approximation $\underline{\phi}$ by increasing, for at least one bit-vector variable, the number of Boolean variables encoding it. Specifically, the new size is implied by the value of this variable in the satisfying assignment to $\bar{\phi}$. This process is repeated until the original formula is shown to be either satisfiable or unsatisfiable. The algorithm is trivially guaranteed to terminate due to the finite domain.

This approach has the potential of being efficient in one of the following two scenarios:

1. The bit-vector formula is satisfiable, and a solution can be represented with a small number of bits.

2. The bit-vector formula is unsatisfiable, and a relatively small number of terms in this formula participate in the proof (i.e., the proof still holds after replacing the other terms with inputs).

Whether this potential is fulfilled depends on one's ability to find such small solutions and small unsatisfiable cores 1 efficiently: For the former, we search for gradually increasing solutions in terms of the number of bits that are needed in order to represent them, and hence are guaranteed to find a small one if it exists; For the latter, modern SAT solvers are quite apt at finding small cores when they exist. In practice, as our experiments show, one of these conditions frequently holds and we are able to detect it with our tool faster than analyzing the formula head-on without any approximations.

Our approach can be seen as an adaptation to bit-vector formulas of our previous work [5] on abstraction-refinement of quantifier-free Presburger Arithmetic,

\footnotetext{
${ }^{1}$ A small unsatisfiable core of the CNF encoding of a formula does not necessarily imply that a small number of terms from the original formula are necessary for the proof, but obviously the two measures are correlated.
} 
which, in turn, was inspired by the proof-based abstraction-refinement approach to model checking proposed by McMillan and Amla [6]. Other than the different problem domain (bit-vectors vs. Presburger formulas), we also extend the theoretical framework to operate on an arbitrary circuit representation of the formula, rather than on a CNF representation. We also employ optimizations specific to bit-vector arithmetic. On the applied side, we report experimental results on a set of benchmarks generated in both hardware and software verification. Our experiments suggest that our approach can be considerably more efficient than directly invoking the SAT solver on the original formula as well as other state-of-the-art decision procedures.

Related Work. Current decision procedures for bit-vector arithmetic fall into one of three categories:

1) Bit-blasting and its variants: Many current decision procedures are based on bit-blasting the input formula to SAT, with a view of handling arbitrary bit-vector operations. The Cogent [7] procedure mentioned earlier belongs to this category. The most current version of CVC-Lite 8 pre-processes the input formula using a normalization step followed by equality rewrites before finally bit-blasting to SAT. Wedler et al. 9 have a similar approach wherein they normalize bit-vector formulas in order to simplify the generated SAT instance. STP [10] is the successor to the CVC-Lite system; it performs several array optimizations, as well as arithmetic and Boolean simplifications on the bit-vector formula before bit-blasting to MiniSat. Yices [1] applies bit-blasting to all bitvector operators except for equality.

2) Canonizer-based procedures: Earlier work on deciding bit-vector arithmetic centered on using a Shostak-like approach of using a canonizer and solver for that theory. The work by Cyrluk et al. 12 and by Barrett et al. on the Stanford Validity Checker [13] fall into this category; the latter differs from the former in the choice of a canonical representation. These approaches are very elegant, but are restricted to a subset of bit-vector arithmetic comprising concatenation, extraction, and linear equations (not inequalities) over bit-vectors.

3) Procedures for modular and bounded arithmetic: The third category of systems focuses on techniques to handle (linear and non-linear) modular arithmetic. The most recent work in this area is by Babić and Musuvathi [14, who encode non-linear operations as non-linear congruences and make novel use of Newton's p-adic method for solving them. However, this approach does not treat some of the operations that we handle such as integer division, and seems harder to extend to new operations. Brinkmann and Drechsler 15] use an encoding of linear bit-vector arithmetic into integer linear programming with bounded variables in order to decide properties of RTL descriptions of circuit data-paths, but do not handle any Boolean operations. Parthasarathy et al. 16 build on this approach by using a lazy encoding with a modified DPLL search, but nonlinear bit-vector arithmetic is not supported. Huang and Cheng 17] give an approach to solving bit-vector arithmetic based on combining ATPG with a solver for linear modular arithmetic. This approach is limited in its treatment of 
non-linear operations which it handles by heuristically rewriting them as linear modular arithmetic constraints.

McMillan and Amla 6] use a technique related to ours in order to accelerate model checking algorithms over finite Kripke structures. Specifically, they invoke a bounded model checker to decide which state variables should be made visible in order to generate a 'good' abstraction for the next iteration of model checking. Gupta et al. 18] propose a similar model-checking framework, which among others makes greater use of counterexamples and uses abstract models for both validation and falsification attempts. Our approach differs from both of these in the following respects: we use a bit-vector decision procedure instead of a model checker, and we seek to eliminate constraints rather than variables (or gates or latches, as the case may be).

Lahiri et al. 19] present an algorithm for deciding satisfiability of quantifierfree Presburger arithmetic that is based on alternating between an under- and an over-approximation. The under-approximation is constructed as in [5]. The over-approximation uses a Craig Interpolant.

\section{Preliminaries}

Boolean Satisfiability. We assume the reader is familiar with the basic terminology of propositional logic such as resolution, Conjunctive Normal Form and Tseitin encoding 20] (the linear procedure for converting an arbitrary propositional formulas to CNF based on the introduction of a new variable in each node in the DAG representation of the formula).

We remind the reader that SAT solvers can be seen as progressing by performing resolution steps. If the input formula is unsatisfiable, modern SAT solvers such as zChaff [21] and MiniSat [22] can output a proof of unsatisfiability [236] based on resolution. The leafs of such proofs (the assumptions) constitute an unsatisfiable core, i.e., an unsatisfiable subset of the clauses. In practice, SAT solvers tend to find small unsatisfiable cores if they exist. Indeed, in most cases in practice, formulas contain a large amount of redundant constraints.

Bit-Vector Arithmetic. While we are not aware of a standard definition of bit-vector arithmetic (it varies according to needs and tools), the fragment we consider here includes finite-precision integer arithmetic with linear and nonlinear operators, as well as standard bit-wise operators, such as left shift, logical and arithmetic right shifts, extraction, concatenation, and so forth. In fact, the approach we use in this paper is orthogonal to the the set of operators, since it only relies on a given finite width for each variable, as well as on the existence of a propositional encoding of the formula.

At present, UCLID supports the subset of bit-vector arithmetic with the following operators: arbitrary Boolean connectives, relational operators, bitwise Boolean operators, extraction, concatenation, shifts, addition, subtraction, multiplication, division, and modulo.

Each bit-vector expression is associated with a type. The type is the width of the expression in bits and whether it is signed (two's complement encoding) or 
unsigned (binary encoding). Assigning semantics to this language is straightforward, e.g., as done in [15.

Note that all arithmetic operators (addition: + , subtraction: - , multiplication: $*$, division: $\div$, modulo: $\%$ ) are all finite-precision, and come with an associated operator width.

Example 1. The following formula is valid when interpreted over the integers:

$$
(x-y>0) \Longleftrightarrow(x>y)
$$

However, if $x$ and $y$ are interpreted as bit-vectors, this equivalence no longer holds, due to possible overflow on the subtraction operation.

Note also that the relational operators $>,<, \leq, \geq$, the non-linear arithmetic operators $(*, \div, \%)$ and the right-shift depend on whether an unsigned, binary encoding is used or a two's complement encoding is used. We assume that the type of the expression is clear from the context.

This paper addresses the satisfiability problem for bit-vector formulas: given a bit-vector formula $\phi$, is there an assignment to the bits in $\phi$ under which $\phi$ evaluates to True? It is easy to see that this problem is NP-complete.

Notation: We henceforth denote formulas in bit-vector arithmetic as $\phi, \phi^{\prime}, \phi_{1}$, $\phi_{2}, \ldots$, and Boolean formulas as $\beta, \beta_{1}, \beta_{2}, \ldots$

\section{The Decision Procedure}

We now present the main contribution of this paper, a SAT-based decision procedure that operates by generating increasingly precise abstractions of bit-vector formulas. The input to the decision procedure is a bit-vector arithmetic formula $\phi$. Let there be $n$ bit-vector variables appearing in $\phi$, denoted by $\boldsymbol{v}_{1}, \boldsymbol{v}_{2}, \boldsymbol{v}_{3}, \ldots, \boldsymbol{v}_{n}$. Each variable $\boldsymbol{v}_{i}$ has an associated bit-width $w_{i}$.

\subsection{Overview}

We first give a broad overview of our decision procedure, which is illustrated in Figure 1. Details of design decisions are described later in this section.

The decision procedure performs the following steps:

1. Initialization: For each variable $\boldsymbol{v}_{i}$, we select a corresponding number $s_{i}$ of Boolean variables to encode it with, where $0 \leq s_{i} \leq w_{i}$. We will call $s_{i}$ the encoding size of bit-vector variable $\boldsymbol{v}_{i}$.

2. Generate Under-Approximation and Encode to SAT: An under-approximation, denoted $\phi$, is generated by restricting the values of each $\boldsymbol{v}_{i}$ to range over a set of cardinality $2^{s_{i}}$. Thus, the Boolean encoding of $\boldsymbol{v}_{i}$ will comprise $s_{i}$ Boolean variables; note, however, that the length of the vector of Boolean variables replacing $\boldsymbol{v}_{i}$ remains $w_{i}$.

There are several ways to generate such an under-approximation and its Boolean encoding. One option is to encode $\boldsymbol{v}_{i}$ using Boolean variables on its 


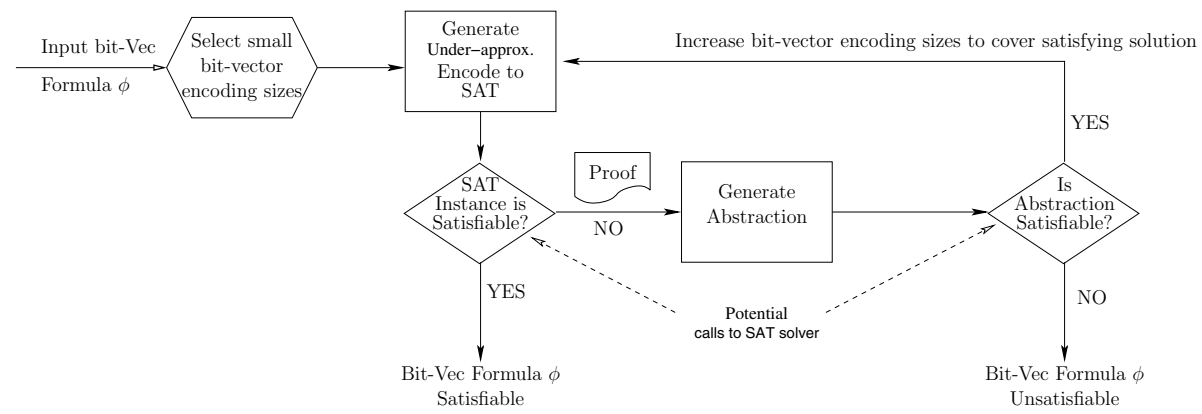

Fig. 1. Abstraction-based approach to solving bit-vector arithmetic

$s_{i}$ low order bits and then zero-extend it to be of length $w_{i}$. The other is to "sign-extend" it instead. For example, if $s_{i}=2$ and $w_{i}=4$, the latter would generate the Boolean vector $\left[\boldsymbol{v}_{i 1}, \boldsymbol{v}_{i 1}, \boldsymbol{v}_{i 1}, \boldsymbol{v}_{i 0}\right]$ (where $\boldsymbol{v}_{i j}$ are Boolean variables). Our implementation currently uses the latter encoding, as it enables searching for solutions at both ends of the ranges of bit-vector values. Further exploration of this aspect is left to future work.

A Boolean formula $\beta$ is then computed from $\phi$ using standard circuit encodings for bit-vector arithmetic operators. The width of the operators is left unchanged. The formula $\beta$ is handed to an off-the-shelf SAT solver. The only feature required of this SAT solver is that its response on unsatisfiable formulas should be accompanied by an unsatisfiable core.

If the SAT solver reports that $\beta$ is satisfiable, then the satisfying assignment is an assignment to the original formula $\phi$, and the procedure terminates. However, if $\beta$ is unsatisfiable, we continue on to the next step.

3. Generate Over-Approximation from Unsatisfiable Core: The SAT solver extracts an unsatisfiable core $\mathcal{C}$ from the proof of unsatisfiability of $\beta$. We use $\mathcal{C}$ to generate an over-approximating abstraction $\bar{\phi}$ of $\phi$. The formula $\bar{\phi}$ is also a bit-vector formula, but typically much smaller than $\phi$.

The algorithm that generates $\bar{\phi}$ is described in Section 3.2 , The key property of $\bar{\phi}$ is that its translation into SAT, using the same sizes $s_{i}$ as those that were used for $\phi$, would also result in an unsatisfiable Boolean formula. The satisfiability of $\bar{\phi}$ is then checked using a sound and complete decision procedure $\mathcal{P}$ for bit-vector arithmetic, e.g., a bit-blasting approach.

If $\bar{\phi}$ is unsatisfiable, we can conclude that so is $\phi$. On the other hand, if $\bar{\phi}$ is satisfiable, it must be the case that at least one variable $\boldsymbol{v}_{i}$ is assigned a value that is not representable with $s_{i}$ Boolean variables (recall the key property enjoyed by $\bar{\phi}$ cited earlier). This larger satisfying assignment indicates the necessary increase in the encoding size $s_{i}$ for $\boldsymbol{v}_{i}$. Proceeding thus, we increase $s_{i}$ for all relevant $i$, and go back to Step 2 .

Remark 1. Note that in this step it would be permissible to merely use a sound, but not necessarily complete, bit-vector arithmetic decision procedure $\mathcal{P}$. In other words, we require that the outcome of $\mathcal{P}$ be correct whenever 
this outcome is 'Unsat', but we can tolerate spurious purported satisfying assignments. Indeed, in cases where $\mathcal{P}$ provides a satisfying assignment that is not a satisfying assignment for $\bar{\phi}$, we can simply increase $s_{i}$ by 1 for each $i$ such that $s_{i}<w_{i}$, and go back to Step 2. Of course, bit-blasting is both sound and complete.

Since $s_{i}$ increases for at least one $i$ in each iteration of this loop, this procedure is guaranteed to terminate in $O\left(n \cdot w_{\max }\right)$ iterations, where $w_{\max }=\max _{i} w_{i}$. Of course, each iteration involves a call to a SAT solver and a decision procedure for bit-vector arithmetic.

One of the main theoretical advances we make over the earlier work on Presburger arithmetic [5] is a different method for generating the abstraction. We describe this in the following section.

\subsection{Generating an Over-Approximating Abstraction}

The earlier work assumed that $\phi$ was in conjunctive normal form (CNF), whereas our procedure works with an arbitrary directed acyclic graph (DAG) or circuitbased representation, which is the format in which the input problems are generated. While $\phi$ can be transformed to CNF (in two different ways, listed below), we argue below that neither of those approaches is desirable, primarily due to the presence of if-then-else (ITE) expressions at arbitrary locations in $\phi$.

1) Eliminating ITE using new variables: By giving each ITE expression in the formula a fresh bit-vector variable name, we can eliminate all ITEs with just a linear blow-up in the formula size. However, this also introduces a number of new bit-vector variables that is linear in the size of the formula.

Note that the number of input bit-vector variables $\left(\boldsymbol{v}_{i}\right.$ 's) is usually a few orders of magnitude smaller than the size of the formula $\phi$. As a result, when treating the new variables as inputs, the SAT solver's performance has been observed to suffer dramatically.

Of course, the values of these new variables are dependent on those of the $\boldsymbol{v}_{i}$ 's, and we can therefore attempt to restrict the SAT solver from case-splitting on the bit-encodings of the new ITE variables. However, such restrictions have also been found to severely adversely affect the run-time of current SAT engines. (It amounts to changing the decision heuristic.)

2) Direct elimination of ITE: Another way of eliminating ITEs is to expand out the cases without introducing new variables. However, this leads to a worst-case exponential blow-up in formula size, which is commonly witnessed in practice.

We have therefore devised an abstraction-generation algorithm $\mathcal{A}$ that operates directly on the DAG representation of $\phi$, denoted $D_{\phi}$. The inputs to $\mathcal{A}$ are $D_{\phi}$, the root node, and the unsatisfiable core $\mathcal{C}$. The output is a DAG $D_{\bar{\phi}}$ representing $\bar{\phi}$, which is an over-approximation of $\phi$. Let $N^{\phi}$ and $N^{\bar{\phi}}$ be the set of nodes in $D_{\phi}$ and $D_{\bar{\phi}}$, respectively.

Before describing the algorithm, we need to describe the process of transforming the Boolean encodings of $\phi$ and $\bar{\phi}$ into CNF. It can be seen as a generalization 
of Tseitin's encoding (which introduces fresh variables for internal nodes) to the case of bit-vector formulas. Each internal node $\mathrm{n} \in N^{\phi}$ is annotated with a set of CNF clauses $c(\mathrm{n})$ that relate the output of that node $o(\mathrm{n})$ to its inputs, according to the operator in the node. These output variables then appear as input to the parent nodes of $\mathrm{n}$. Then a conjunction of the clauses in $\left\{c(\mathrm{n}) \mid \mathrm{n} \in N^{\phi}\right\}$ and one more unit clause with the variable encoding the top node, is the CNF representation of $\phi$. A subset of these clauses constitutes the UNSAT core $\mathcal{C}$. These definitions and notations also apply to $D_{\bar{\phi}}$, and we will use them for both DAGs when the meaning is clear from the context. For a formula (or equivalently a set of clauses) $C$ we denote by $\operatorname{var} s(C)$ the set of variables that appear in $C$.

Procedure $\mathcal{A}$ (see Algorithm 1) recurses down the structure of $D_{\phi}$ and creates $D_{\bar{\phi}}$. It replaces a Boolean node $\mathrm{n}$ with a new variable and backtracks, if and only if none of the variables in $\operatorname{var} s(c(\mathrm{n}))$ are present in $\mathrm{C}^{2}$. It uses the functions left-child $\left(D_{\phi}, \mathrm{n}\right)$ and right-child $\left(D_{\phi}, \mathrm{n}\right)$ to return the left and right child of $\mathrm{n}$ on $D_{\phi}$, respectively.

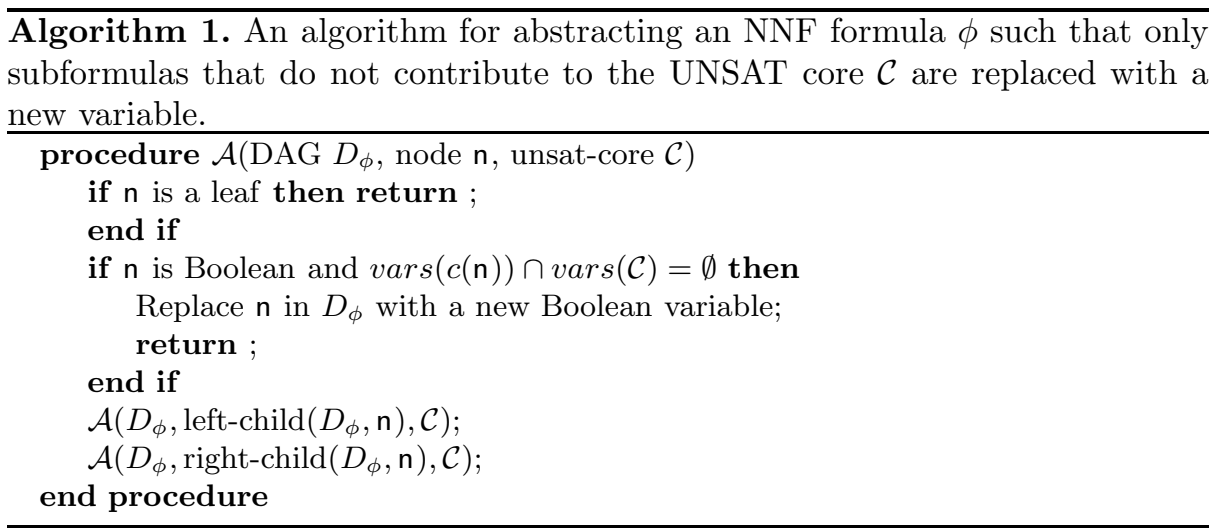

The replacement of Boolean nodes with new variables can be further optimized using the "pure-literal rule": if $\mathrm{n}_{\phi}$ is a Boolean-valued node and only appears unnegated, replace it by True; likewise, if $\mathrm{n}_{\phi}$ only appears negated, replace it by False. In other words, in such cases no new Boolean variable is needed.

Note that the resulting DAG $D_{\bar{\phi}}$ can be embedded into $D_{\phi}$. For each node $\mathrm{n} \in N^{\phi}$ we will denote by $\overline{\mathrm{n}}$ its counterpart in $D_{\bar{\phi}}$ before the abstraction process begins (after the abstraction some of them can be eliminated by simplifications).

The correctness of our abstraction technique is formalized by the following two theorems:

Theorem 1. $\bar{\phi}$ is an over-approximating abstraction of $\phi$.

${ }^{2}$ The same replacement criterion can be applied to bit-vector-valued nodes, which can then be replaced with fresh bit-vector variables. Our implementation ignores this option, however, and we shall therefore also ignore this possibility in the proof. 
Proof. Let $\alpha$ be a satisfying assignment of $\phi$. We show how to construct $\bar{\alpha}$, a satisfying assignment for $\bar{\phi}$. First, for each variable $v \in \operatorname{var} s(\phi)$ such that the (leaf) node representing $v$ is still present in $D_{\bar{\phi}}$, define $\bar{\alpha}(v)=\alpha(v)$. Second, for each Boolean variable $b \in\{\operatorname{var} s(\bar{\phi}) \backslash \operatorname{var} s(\phi)\}$ (i.e., the new abstracting variables) represented by node $\mathrm{n} \in D_{\bar{\phi}}$, define $\bar{\alpha}(b)$ to be equal to the Boolean value of the corresponding node in $D_{\phi}$, as implied by $\alpha$. For example, if $\alpha\left(b_{1}\right)=$ True, $\alpha\left(b_{2}\right)=$ False and the node $b_{1} \vee b_{2}$ was replaced with a new variable $b$, then $\bar{\alpha}(b)=$ True $\vee$ False $=$ True. Clearly, $\bar{\alpha}$ satisfies $\bar{\phi}$, since every node in $D_{\bar{\phi}}$ is evaluated the same as its counterpart in $D_{\phi}$. Hence, if $\phi$ is satisfiable, then so is $\bar{\phi}$, which implies the correctness of the Theorem.

Next, we have to prove termination. Termination is implied if we can show that any satisfying assignment to $\bar{\phi}$ requires width larger than the current one $s_{i}$ (i.e., the width with which the unsatisfiable core $\mathcal{C}$ was derived), or, equivalently:

Theorem 2. The SAT encoding of $\bar{\phi}$ with encoding sizes $s_{i}$ is unsatisfiable.

Proof. We will prove that the CNF encoding of $\bar{\phi}$ with sizes $s_{i}$ contains the clauses of the UNSAT core $\mathcal{C}$.

Three observations about this encoding are important for our proof:

1. First, for an internal node $\mathrm{n}$ that represents a Boolean operator, each clause in $c(\mathrm{n})$ contains the output variable of its node. For example, the CNF of an 'and' node $o=a \wedge b$ is $(o \vee \neg a \vee \neg b),(\neg o \vee a),(\neg o \vee b)$, and indeed $o$, the output variable of this node, is present in all three clauses. The same applies to the other Boolean operators. Hence, we can write $o(c l)$ for a clause $c l$ to mean the output variable of the node that $c l$ annotates (hence, $o(c l) \in c l$ ).

2. Second, the same observation applies to predicates over bit-vectors. For simplicity, we concentrate only on the bit-vector equality predicate. In such a node, each clause contains either the output variable or an auxiliary variable present only in this node. For example, the CNF of the node $o=\left(\boldsymbol{v}_{\mathbf{1}}=\boldsymbol{v}_{\mathbf{2}}\right)$ for 2-bit bit-vectors $\boldsymbol{v}_{\mathbf{1}}$ and $\boldsymbol{v}_{\mathbf{2}}$, is the following (the first four clauses encode $x=\left(\boldsymbol{v}_{\mathbf{1}}[0]=\boldsymbol{v}_{\mathbf{2}}[0]\right)$, the other clauses encode $o=x \wedge\left(\boldsymbol{v}_{\mathbf{1}}[1]=\boldsymbol{v}_{\mathbf{2}}[1]\right)$ where $x$ is the local auxiliary variable):

$$
\begin{aligned}
& \left(x \vee \boldsymbol{v}_{\mathbf{1}}[0] \vee \boldsymbol{v}_{\mathbf{2}}[0]\right),\left(x \vee \neg \boldsymbol{v}_{\mathbf{1}}[0] \vee \neg \boldsymbol{v}_{\mathbf{2}}[0]\right), \\
& \left(\neg x \vee \boldsymbol{v}_{\mathbf{1}}[0] \vee \neg \boldsymbol{v}_{\mathbf{2}}[0]\right),\left(\neg x \vee \neg \boldsymbol{v}_{\mathbf{1}}[0] \vee \boldsymbol{v}_{\mathbf{2}}[0]\right), \\
& \left(o \vee \neg x \vee \neg \boldsymbol{v}_{\mathbf{1}}[1] \vee \neg \boldsymbol{v}_{\mathbf{2}}[1]\right),\left(o \vee \neg x \vee \boldsymbol{v}_{\mathbf{1}}[1] \vee \boldsymbol{v}_{\mathbf{2}}[1]\right), \\
& \left(\neg O \vee \boldsymbol{v}_{\mathbf{1}}[1] \vee \neg \boldsymbol{v}_{\mathbf{2}}[1]\right),\left(\neg O \vee \neg \boldsymbol{v}_{\mathbf{1}}[1] \vee \boldsymbol{v}_{\mathbf{2}}[1]\right),(\neg O \vee x)
\end{aligned}
$$

3. Finally, observe that resolution among clauses that relate the output and input of a node using the output variable as the resolution variable, results in a tautology. For example, recall the CNF representation of the 'and' node above: Resolving on the output variable $o$ of that node results in a tautology. The same observation applies to other Boolean operators and equality between bit-vectors. 
We use these observations for analyzing the three possible cases for a node $\mathrm{n}$ in $D_{\phi}$ : either it is retained in $D_{\bar{\phi}}$, replaced with a new variable, or eliminated. Our goal, recall, is to show that despite the abstraction implied by these changes to the DAG, the set of clauses that encode the new DAG $D_{\bar{\phi}}$ still contains the UNSAT core $\mathcal{C}$ of $\phi$.

- Claim: for each node $\mathrm{n} \in N^{\phi}$ for which the corresponding $\overline{\mathrm{n}} \in N^{\bar{\phi}}$ is retained in the abstraction process, $c(\mathrm{n}) \cap \mathcal{C}=c(\overline{\mathrm{n}}) \cap \mathcal{C}$.

Proof. Since $\mathrm{n}$ and $\overline{\mathrm{n}}$ encode the same operator and receive the same type of input (e.g., if $\mathrm{n}$ and $\overline{\mathrm{n}}$ represent a bit-vector operator, then their respective inputs are bit-vectors of the same width), then $c(\mathbf{n})$ and $c(\overline{\mathrm{n}})$ are equivalent up to renaming of variables. Such a renaming can occur if the abstraction process replaced one of the inputs (or both) with a new variable. But this means that none of these inputs are in $\mathcal{C}$, hence those clauses in $c(\overline{\mathrm{n}})$ that contain renamed literals, are not in $\mathcal{C}$. Hence, $c(\mathrm{n}) \cap \mathcal{C}=c(\overline{\mathrm{n}}) \cap \mathcal{C}$.

- Claim: for each node $\mathrm{n} \in N^{\phi}$ that was replaced with a new variable in $N^{\bar{\phi}}$, $c(\mathrm{n}) \cap \mathcal{C}=\emptyset$.

Proof. This is trivial by the construction of the abstraction: if any of the clauses in $c(\mathbf{n})$ were in $\mathcal{C}$, then this node would not be replaced with a new variable.

- Claim: For each node $\mathrm{n} \in N^{\phi}$ whose corresponding node $\overline{\mathrm{n}} \in N^{\bar{\phi}}$ was eliminated (i.e., the paths of this node to the root were all 'cut' by the abstraction), $c(\mathrm{n}) \cap \mathcal{C}=\emptyset$.

Proof. On each path from $\mathrm{n}$ to the root node, there exists one or more nodes other than $\mathrm{n}$ that were replaced with free variables. For simplicity of the proof, we will consider one such path and denote the closest node to $\mathrm{n}$ that was replaced with a new variable by $\mathrm{n}_{c}$.

We will now prove the claim by induction on the distance (in terms of number of DAG operators) from $\mathrm{n}$ to $\mathrm{n}_{c}$. In the base case $\mathrm{n}$ is a direct child of $\mathrm{n}_{c}$. Falsely assume that there exists a clause $c l \in c(\mathrm{n})$ such that $c l \in \mathcal{C}$. $c\left(\mathrm{n}_{c}\right)$ contain $o(\mathrm{n})$, the output variable of $\mathrm{n}$, and $c l$ also contains $o(\mathrm{n})$ (see observations 1 and 2 above). Hence, if $c l \in \mathcal{C}$, then $o(\mathrm{n}) \in \operatorname{var} s(\mathcal{C})$ which contradicts the condition for abstracting $\mathrm{n}_{c}$ with a new variable.

For the induction step falsely assume that there exists a clause $c l \in c(\mathbf{n})$ such that $c l \in \mathcal{C}$. By the induction hypothesis, none of the clauses in the parent node of $n$ are in $\mathcal{C}$. Hence, only clauses from $c(\mathrm{n})$ can contain the output variable of $\mathrm{cl}$ in $\mathcal{C}$. This means that $o(\mathrm{cl})$ can only be resolved-on among $c(\mathrm{n})$ clauses. By noting that that this kind of resolution can only result in a tautology (see observation 3 above), this resolution step cannot be on the path to the empty clause in the resolution proof. This contradicts, however, the requirement that any variable in every clause that participates in a proof of the empty clause must be resolved on in order to eliminate it.

Thus, the set of clauses annotating $D_{\bar{\phi}}$ contains $\mathcal{C}$ and hence $\bar{\phi}$ is unsatisfiable. 
In comparison with our previous CNF-based abstraction scheme [5], we note that, for ITE-free formulas, that approach can generate more compact abstractions, as they do not introduce new variables. However, for real-world benchmarks from both hardware and software verification, such as those discussed in the following section, we found that elimination of ITEs leads to significant space and time overheads. The approach of this paper allows us to extend the abstraction-based approach to operate on arbitrary DAG-like formulas. Moreover, we have found that the Boolean structure in the original bit-vector formula is not usually the primary source of difficulty; it is the bit-vector constraints that are the problem.

\subsection{Another Step of Abstraction}

It is well-known that certain bit-vector arithmetic operators, such as integer multiplication of two variables (of adequately large width), are extremely hard for a procedure based on bit blasting. However, for many problems involving these operators, it is unnecessary to reason about all of the operators' properties in order to decide the formula. Instead, using a set of rules (based on wellknown rewrite rules) allows us to perform fine-grained abstractions of functions, which often suffices. Such (incomplete) abstractions can be used in the overapproximation phase of our procedure, while maintaining the overall procedure sound and complete (see Remark 1 in \$3.1). This is a major advantage, because these rules can be very powerful in simplifying the formula.

Therefore, UCLID invokes a preprocessing step before calling Algorithm $\mathcal{A}$. In this step, it replaces a subset of "hard" operators by lambda expressions that partially interpret those operators. The resulting formula is then bit blasted to SAT.

For example, UCLID replaces the multiplication operator $*_{w}$ of width $w$ (for $w>4$, chosen according to the capacity of current SAT engines) by the following lambda expression involving the freshly introduced uninterpreted function symbol $\mathrm{mul}_{w}$ :

$$
\lambda x \cdot \lambda y \cdot \operatorname{ITE}\left(x=0 \vee y=0,0, \operatorname{ITE}\left(x=1, y, \operatorname{ITE}\left(y=1, x, \operatorname{mul}_{w}(x, y)\right)\right)\right)
$$

This expression can be seen as partially interpreting multiplication, as it models precisely the behavior of this operator when one of the arguments is 0 or 1 , but is uninterpreted otherwise.

\section{Experimental Results}

The new procedure is now incorporated within the UCLID verification system [24], which is implemented in Moscow ML 25] (a dialect of Standard ML). MiniSat 22] was used as the SAT solver to solve over-approximations, while Booleforce (written by Armin Biere) was used as a proof-generating SAT solver for under-approximations. The initial value of $s_{i}$ is set to $\min \left(4, w_{i}\right)$ for benchmarks not involving hard operators (like multiplication) while it is set to $\min \left(2, w_{i}\right)$ otherwise. 
Table 1. Comparison of run-time of abstraction-based approach (UCLID) with bit-blasting, STP, Yices. The best run-time is highlighted in bold font. A "TO" indicates a timeout of 3600 seconds was reached. An "err" indicates that the solver could not handle bit-vectors of width as wide as those in the benchmark or quit with an exception. Bit-blasting used MiniSat. UCLID used Booleforce for proof generation and MiniSat on the abstraction. STP is based on MiniSat. "Ans" indicates whether the formula was satisfiable (SAT) or not (UNSAT). "Enc" indicates time for translation to SAT, and "SAT" indicates the time taken by the SAT solver (both calls).

\begin{tabular}{|c|c|c|c|c|c|c|c|c|c|}
\hline \multirow{3}{*}{ Formula } & \multirow{3}{*}{ Ans. } & \multirow{2}{*}{\multicolumn{3}{|c|}{$\begin{array}{c}\text { Bit-Blasting } \\
\text { Run-time (sec.) }\end{array}$}} & \multirow{2}{*}{\multicolumn{3}{|c|}{$\begin{array}{c}\text { UCLID } \\
\text { Run-time (sec.) }\end{array}$}} & \multirow{3}{*}{$\begin{array}{r}\text { STP } \\
\text { (sec.) }\end{array}$} & \multirow{3}{*}{$\begin{array}{l}\text { Yices } \\
\text { (sec.) }\end{array}$} \\
\hline & & & & & & & & & \\
\hline & & Enc. & SAT & Total & Enc. & SAT & Total & & \\
\hline Y86-std & UNSAT & 17.91 & TO & $\mathrm{TO}$ & 23.51 & 987.91 & 1011.42 & 2083.73 & TO \\
\hline Y86-btnft & UNSAT & 17.79 & TO & TO & 26.15 & 1164.07 & $1190.22 \mid$ & err & TO \\
\hline s-40-50 & SAT & 6.00 & 33.46 & 39.46 & 106.32 & 10.45 & 116.77 & 12.96 & 65.51 \\
\hline BBB-32 & SAT & 37.09 & 29.98 & 67.07 & 19.91 & 1.74 & 21.65 & 38.45 & 183.30 \\
\hline rfunit_flat-64 & SAT & 121.99 & 32.16 & 154.15 & 19.52 & 1.68 & 21.20 & 873.67 & 1312.00 \\
\hline C1-P1 & SAT & 2.68 & 45.19 & 47.87 & 2.61 & 0.58 & 3.19 & err & err \\
\hline C1-P2 & UNSAT & 0.44 & TO & $\mathrm{TO}$ & 2.24 & 2.12 & 4.36 & TO & TO \\
\hline C3-OP80 & SAT & 14.96 & TO & TO & 14.54 & 349.41 & 363.95 & TO & 3242.43 \\
\hline egt-5212 & UNSAT & 0.064 & 0.003 & 0.067 & 0.163 & 0.001 & 0.164 & 0.018 & 0.009 \\
\hline
\end{tabular}

Table 1 shows experimental results obtained on a set of bit-vector formulas. We compare the run-time of UCLID against bit blasting to MiniSat, and the STP [10] and Yices [11] decision procedures. (The latter two procedures jointly won the bit-vector division of the recent SMT-COMP'06 competition, and we compare against the versions that were entered in the competition.) All results were obtained on a system with a $2.8 \mathrm{GHz}$ Xeon processor and $2 \mathrm{~GB}$ RAM. The benchmarks are drawn from a wide range of sources, arising from verification and testing of both hardware and software 3

- Verification of word-level versions of an x86-like processor model [26] (Y86std, Y86-btnft);

- Detection of format-string vulnerabilities in C programs 27] (s-40-50);

- Hardware verification benchmarks obtained from Intel, slightly modified (BBB-32, rfunit_flat-64);

- Word-level combinational equivalence checking benchmarks obtained from a CAD company (C1-P1, C1-P2, C3-OP80); and

- Directed random testing of programs [10 (egt-5212). This represents the set of benchmarks used in SMT-COMP'06, which are easily solved within a fraction of a second. (As the run-times on this benchmark was so small, we state them to three decimal places, unlike the others.)

\footnotetext{
${ }^{3}$ All benchmarks that we have permission to make publicly available are online at http://www.cs.cmu.edu/ uclid/tacas07-examples.tgz

${ }^{4}$ Name withheld on their request.
} 
Table 2. Statistics on the abstraction-based approach (UCLID). " $\max _{i} s_{i}$ " indicates the maximum value of $s_{i}$ generated in the entire run. "Num. Iter" indicates the number of iterations of the abstraction-refinement loop where an iteration is counted if at least one of the SAT solver calls is made. The second to last column compares the size of the largest abstraction $\bar{\phi}$ created as a fraction of the size of the original formula $\phi$, where sizes are measured as the number of nodes in the DAG representations of the formulas. "Speedup" indicates the factor by which the abstraction-based approach is faster than its nearest competitor, or slower than the best solver.

\begin{tabular}{|c|c||r|r|r|c|r|}
\hline Formula & Ans. & $\max _{i} s_{i}$ & $\max _{i} w_{i}$ & Num. Iter & $\max \frac{|\bar{\phi}|}{|\phi|}$ & Speedup \\
\hline Y86-std & UNSAT & 4 & 32 & 1 & 0.18 & 2.06 \\
Y86-btnft & UNSAT & 4 & 32 & 1 & 0.20 & $>3.01$ \\
s-40-50 & SAT & 32 & 32 & 8 & 0.12 & 0.11 \\
BBB-32 & SAT & 4 & 32 & 1 & - & 1.78 \\
rfunit_flat-64 & SAT & 4 & 64 & 1 & - & 7.27 \\
C1-P1 & SAT & 2 & 65 & 1 & - & 15.00 \\
C1-P2 & UNSAT & 2 & 14 & 1 & 1.00 & 825.69 \\
C3-OP80 & SAT & 2 & 9 & 1 & - & 8.91 \\
egt-5212 & UNSAT & 8 & 8 & 1 & 0.13 & 0.06 \\
\hline
\end{tabular}

The first three sets of benchmarks involve only (finite-precision) linear arithmetic. The combinational equivalence checking benchmarks involve finite-precision multiplication with large widths (e.g., C1-P1 and C1-P2 involve 65-bit, 49-bit, and 30-bit multiplication), apart from bitwise operations including extraction and concatenation. The last set includes linear arithmetic and bitwise operations.

An analysis of UCLID's performance on the benchmarks is given in Table 2 We observe the following: 1) Only very few iterations of the abstraction-refinement loop are required, just 1 in most cases; 2) The abstractions generated are small in most cases; and 3) UCLID yields a speed-up in all but one case when the number of iterations is 1 . In the 2 other cases, where some $s_{i}$ reached the maximum $w_{i}$, it performs worse.

We look more closely at two benchmarks. UCLID's performance is orders of magnitude better than the other solvers on the C1-P2 benchmark: this involves multiplication as noted earlier, and the abstraction described in Section 3.3 was particularly effective. However, on the benchmark s-40-50, it is 10 times worse than STP, with most of the time spent in encoding. This problem is mainly due to re-generation of the SAT instance in each step, which an incremental implementation can fix.

The results indicate a complementarity amongst the solvers with respect to this set of benchmarks: either bit-blasting (with rewrites as explained in \$3.3) is effective, or the problem is unsatisfiable with a small UNSAT core, or there is a satisfying solution within a small range at the high and low ends of the bitvector's value domain. In the latter two cases, our abstraction-based approach is effective. 


\section{Conclusion}

We have demonstrated the utility of an abstraction-based approach for deciding the satisfiability of finite-precision bit-vector arithmetic. The speed-ups we have obtained, especially on benchmarks involving non-linear arithmetic operations, indicate the promise of the proposed approach. The algorithm is applicable in many areas in formal verification (e.g., word-level bounded model checking) and can be extended to handle floating-point arithmetic. Ongoing and future work includes generalizing the form of over- and under-approximations beyond those we have proposed herein, and making the encoding to SAT incremental.

\section{References}

1. Biere, A., Cimatti, A., Clarke, E., Yhu, Y.: Symbolic model checking without BDDs. In: TACAS. (1999) 193-207

2. Clarke, E., Kroening, D.: Hardware verification using ANSI-C programs as a reference. In: Proceedings of ASP-DAC 2003, IEEE Computer Society Press (2003) 308-311

3. Xie, Y., Aiken, A.: Scalable error detection using Boolean satisfiability. In: Proc. 32nd ACM Symposium on Principles of Programming Languages (POPL). (2005) 351-363

4. Arons, T., Elster, E., Fix, L., Mador-Haim, S., Mishaeli, M., Shalev, J., Singerman, E., Tiemeyer, A., Vardi, M.Y., Zuck, L.D.: Formal verification of backward compatibility of microcode. In: Proc. Computer-Aided Verification (CAV'05). LNCS 2404 (2005) 185-198

5. Kroening, D., Ouaknine, J., Seshia, S.A., Strichman, O.: Abstraction-based satisfiability solving of Presburger arithmetic. In: Proc. CAV. Volume 3114 of LNCS. (2004) 308-320

6. McMillan, K., Amla, N.: Automatic abstraction without counterexamples. In: Proceedings of TACAS 03. Volume 2619., Springer LNCS (2003) 2-17

7. Cook, B., Kroening, D., Sharygina, N.: Cogent: Accurate theorem proving for program verification. In: Proceedings of CAV 2005, Springer (2005) 296-300

8. Berezin, S., Ganesh, V., Dill, D.: A decision procedure for fixed-width bit-vectors. Technical report, Computer Science Department, Stanford University (2005)

9. Wedler, M., Stoffel, D., Kunz, W.: Normalization at the arithmetic bit level. In: Proc. DAC, ACM Press (2005) 457-462

10. Cadar, C., Ganesh, V., Pawlowski, P.M., Dill, D.L., Engler, D.R.: EXE: Automatically generating inputs of death. In: 13th ACM Conference on Computer and Communications Security (CCS '06), ACM (2006) 322-335

11. Dutertre, B., de Moura, L.: The Yices SMT solver. Available at http://yices.csl.sri.com/tool-paper.pdf (2006)

12. Cyrluk, D., Möller, M.O., Rueß, H.: An efficient decision procedure for the theory of fixed-sized bit-vectors. In: Computer-Aided Verification (CAV '97). (1997) 60-71

13. Barrett, C.W., Dill, D.L., Levitt, J.R.: A decision procedure for bit-vector arithmetic. In: Proceedings of DAC'98, ACM Press (1998) 522-527

14. Babić, D., Musuvathi, M.: Modular Arithmetic Decision Procedure. Technical report, Microsoft Research, Redmond (2005)

15. Brinkmann, R., Drechsler, R.: RTL-datapath verification using integer linear programming. In: Proceedings of VLSI Design. (2002) 741-746 
16. Parthasarathy, G., Iyer, M.K., Cheng, K.T., Wang, L.C.: An efficient finite-domain constraint solver for circuits. In: Design Automation Conference (DAC). (2004) $212-217$

17. Huang, C.Y., Cheng, K.T.: Assertion checking by combined word-level ATPG and modular arithmetic constraint-solving techniques. In: Proc. DAC. (2000) 118-123

18. Gupta, A., Ganai, M., Yang, Z., Ashar, P.: Iterative abstraction using SAT-based BMC with proof analysis. In: ICCAD. (2003)

19. Lahiri, S., Mehra, K.: Interpolant based decision procedure for quantifier-free Presburger arithmetic. Technical Report 2005-121, Microsoft Research (2005)

20. Tseitin, G.: On the complexity of proofs in poropositional logics. In Siekmann, J., Wrightson, G., eds.: Automation of Reasoning: Classical Papers in Computational Logic 1967-1970. Volume 2., Springer-Verlag (1983) Originally published 1970.

21. zChaff. http://www.ee.princeton.edu/ ${ }^{\sim}$ chaff/zchaff.php.

22. MiniSat. http://www.cs.chalmers.se/Cs/Research/FormalMethods/MiniSat/.

23. Zhang, L., Malik, S.: Extracting small unsatisfiable cores from unsatisfiable Boolean formulas. In: Proceedings of SAT 03. (2003)

24. UCLID verification system. http://www.cs.cmu.edu/ uclid.

25. Moscow ML. http://www.dina.dk/ sestoft/mosml.html.

26. Bryant, R.E.: Term-level verification of a pipelined CISC microprocessor. Technical Report CMU-CS-05-195, Computer Science Department, Carnegie Mellon University (2005)

27. Wisconsin Safety Analyzer Project. http://www.cs.wisc.edu/wisa. 